\title{
PINK RIEFENSTAHL. (UN-)AUTHENTISCHE INSZENIERUNGEN VON HOMOPHILIE UND HOMOPHOBIE IM MUSIKVIDEO $\gg$ MANN GEGEN MANN« VON RAMMSTEIN
}

\author{
Christian Diemer
}

\section{Hintergrund}

An kaum einer Band spitzen sich Fragen von Authentizität und Inszenierung derart polar zu wie an Rammstein. Erst in der Verschränkung beider Begriffe (als inszenierter Authentizität bzw. authentischer Inszenierung) lassen sich die meisten Meinungsbeiträge zur Rammstein-Rezeption und zum Rammstein-Diskurs überhaupt verstehen - radikal verwerfende ebenso wie emphatisch in Schutz nehmende, auch die Einlassungen der Bandmitglieder selbst, etwa in Interviews. Gleiches gilt für die meisten Songs und Musikvideos der Band.

Authentizität ist im Fall Rammsteins an Provokation, ans »Ärgermachen « gekoppelt. ${ }^{1}$ Gerade weil die Songtexte und -inhalte bisweilen massiv anecken, werden sie von den Rammstein-Anhängern als besonders authentisch, als dem herrschenden System politischer correctness und der marktwirtschaftlichen Stromlinienförmigkeit unangepasst verteidigt. ${ }^{2}$ Verteidigt werden sie implizit oder explizit gegen diejenigen, die wiederum aufgrund derselben Textinhalte schließen, dass es sich bei der Band um eine Truppe Rechter, Perverser oder schlicht hemmungslos Unverantwortlicher handele. Auch und gerade die radikale Negativbewertung vieler Kritiker gründet darauf, dass die Texte entweder in ihren Aussagen ernst genommen werden, oder dass ihre von Band und Fans immer wieder unterstrichene Bedenken-

1 Vgl. Schneider 2001: 26; Landers 2001: 34; sowie mit Verweis auf die Sozialisation der Bandmitglieder in der DDR Kruspe 2001: 20; Lindemann 2001: 33.

2 Zu den Bedeutungsspektren und -ebenen des Begriffs Authentizität vgl. u.a. Knaller/Müller 2006. 
losigkeit und >Unernsthaftigkeit ‘ zwar anerkannt, aber erst recht als inakzeptabel kritisiert wird.

Dies führt zu den in immer neuer Auflage fruchtlos ausgetragenen Kontroversen über die immer ähnlichen Streitpunkte, etwa ob Text, Musik und Inszenierung der Band als rechts oder unpolitisch (oder gar links) zu bewerten sind (vgl. Bettendorf o.J.: 82-84), ob (sexuelle) Gewalt und Katastrophen verherrlicht oder vielmehr dem ihnen sonst auferlegten gesellschaftlichen Tabu entrissen werden etc. Die Tatsache, dass diese Kontroversen weder zu einem Konsens führen noch anderweitig Erkenntnisgewinn abwerfen, legt nahe, dass sie den Kern des Phänomens >Rammstein< nicht oder nur unzulänglich treffen. Nur unter der Kategorie >Authentizität< betrachtet, ergibt sich für die artistischen (vermeintlichen) Diskussionsbeiträge Rammsteins ein inkonsistentes, von den jeweiligen Gegenseiten zu Recht beanstandetes Bild. Aus der Betrachtung der von Rammstein geschaffenen »Gesamtkunstwerke (Hof 2001: 3) hinwiederum lässt sich die Frage, ob die Band und ihre Mitglieder als Personen tatsächlich und glaubhaft rechts oder links sind, überhaupt nicht beantworten.

Versteht man ihre Stücke dagegen als inszenierte Darbietungen, so wird eine weitaus differenziertere und aufschlussreichere Einsicht möglich. Bei entsprechender Betrachtung bieten sich die Rammstein-Songs dar als komplexe Angebote höchst unterschiedlicher Sinnkonstruktionen und Sinndekonstruktionen, die zueinander in widersprüchlichen Verhältnissen stehen und bisweilen auf ausgeklügelte Weise in einem Gemenge von Indifferenz und Irritation zusammenwirken. In dem Maße, in dem sich der Fokus verschiebt von einem (normativ) moralischen zu einem (normativ) ästhetischen (Un-)Authentizitätsbegriff, wird eine Entkoppelung der konkurrierenden Authentizitäts-Anliegen möglich, individuell-situative stimmigkeit trennt sich von moralischer Verallgemeinerbarkeit (vgl. Knaller/Müller 2006: 8/10). Die Inkonsistenz der aus dem ausschließlichen Fokus auf (moralisch) >authentische< Meinungsäußerung erwachsenen, einander diametral entgegen stehenden Bewertungen Rammsteins lässt sich auflösen und als ästhetische Wirkungsstrategie entschlüsseln.

Der vorliegende Aufsatz versucht, dieses am Beispiel des Musikvideos »Mann gegen Mann« aus dem Album Rosenrot in einer Detailanalyse nachzuvollziehen. 


\section{Analyse}

Das Lied »Mann gegen Mann« ist der zweite Titel des 2005 produzierten und am 28. Oktober 2005 erschienenen Albums Rosenrot (Rammstein 2005). Jonas Åkerlund führte Regie für das Video der Single-Auskoppelung (Rammstein 2006; vgl. Fuchs-Gamböck/Schatz 2010: 175/179).

\subsection{Text}

Rammstein-Gitarrist Paul Landers äußert sich zum Text von »Mann gegen Mann $\ll$ :

»Wenn man die Texte [des Albums] gedruckt liest und sie des durch den Vortrag bedingten Bedrohlichen beraubt sind, stellt man fest, dass gerade >Mann gegen Mann eine ganz süße Lyrik über Schwule ist. In unserem Zusammenhang wirkt das fast wie das Gegenteil. Das finden wir aber gut. Da kann jeder gucken, wie locker er ist, das ist ein kleiner Test!« (zit. n. FuchsGamböck/Schatz 2010: 85).

Bassist Oliver Riedel erklärt:

»Wir haben den Song vorher einigen Schwulen vorgespielt, und die fanden den Titel ganz gut! [...] Wir hatten auf jeden Fall nicht vor, eine SchwulenHymne zu schreiben. Natürlich ist es ein kritisches Thema, wie ein heterosexueller Mensch mit dem Thema homosexueller Mensch umgeht. Vielleicht können wir dazu beitragen, dass das Wort `Schwuler` etwas entschärft und der negative Touch relativiert wird « (zit. n. ebd.: 85).

Im Jugendmagazin Zünder der Wochenzeitschrift Zeit wird »Mann gegen Mann« als »homoerotische Fantasie in Moll« charakterisiert, deren »Message« sei: »Schwulsein ist auch irgendwie okay« (zit. n. ebd.: 156).

Die drei zitierten Aussagen führen mitten in die Kernfrage zum Verständnis - des Textes und, wie sich zeigen wird, auch der Musik und des Videos: Ist der Song ein Plädoyer für oder ein Hasslied gegen Schwule? Der Text macht gar nicht von Anfang an klar, dass es überhaupt um Schwule geht. Einige der Sprachbilder sind hinsichtlich ihrer inhaltlichen Aussagekraft ziemlich artifiziell und verbleiben teilweise in surrealer Schwebe. 
Rammstein: »Mann gegen Mann«

Das Schicksal hat mich angelacht und mir ein Geschenk gemacht, warf mich auf einen warmen Stern, der Haut so nah, dem Auge fern.

Ich nehm' mein Schicksal in die Hand, mein Verlangen ist bemannt.

Wo das süße Wasser stirbt, weil es sich im Salz verdirbt, trag ich den kleinen Prinz im Sinn, ein König ohne Königin.

Wenn sich an mir ein Weib verirrt, dann ist die Hölle weltverwirrt.

Mann gegen Mann! Meine Haut gehört dem Herrn.

Mann gegen Mann! Gleich und Gleich gesellt sich gern.

Mann gegen Mann! Ich bin der Diener zweier Herrn.

Mann gegen Mann! Gleich und Gleich gesellt sich gern.

Ich bin die Ecke aller Räume, ich bin der Schatten aller Bäume.

In meiner Kette fehlt kein Glied, wenn die Lust von hinten zieht.

Mein Geschlecht schimpft mich Verräter.

Ich bin der Alptraum aller Väter.

Mann gegen Mann! Meine Haut gehört dem Herrn.

Mann gegen Mann! Gleich und gleich gesellt sich gern.

Mann gegen Mann! Doch friert mein Herz an manchen Tagen.

Mann gegen Mann! Kalte Zungen, die da schlagen,

kalte Zungen, die da schlagen:

Schwule! Ah! Schwule! Ah!

Mich int'ressiert kein Gleichgewicht, mir scheint die Sonne ins Gesicht.

Doch friert mein Herz an manchen Tagen.

Kalte Zungen, die da schlagen:

Schwule! Mann gegen Mann!

Schwule! Mann gegen Mann!

Schwule! Mann gegen Mann!

Schwule! Mann gegen Mann gegen Mann! 
Explizit wird es erst spät, nach dem zweiten Refrain: »Schwule!« Die Bewertung ist durch die zugesetzten Schreie »Ah!«m.E. klar negativ: Um einen eklen Ausruf handelt es sich, ausgestreckten Fingers gewissermaßen. Alternativ ließe sich an einen Aufschrei des Schmerzes oder einen Hilferuf denken, entweder seitens eines Opfers homophober Gewalt, oder seitens eines Homophoben, dem die Konfrontation mit Homosexualität geradezu physischen Schmerz bereitet. Abhängig von der jeweiligen Zuordnung lassen sich diese Erweiterungen des Refrains auch als Dialoge verstehen, einer ruft: »Schwule! «, der andere antwortet »Ah! « Es fällt mir schwer, in dem »Ah!« einen Lustschrei zu erkennen, aber auch diese Deutung kann aus der textlichen Verfasstheit heraus nicht ausgeschlossen werden.

Nächst explizit sind die zwei auf den Refrain hinführenden Statements. Gegenüber alltagssprachlichen Situationen und Redeweisen anschlussfähig, geben sie einen relativ unverschlüsselten Wink in die richtige Richtung:

»Wenn sich an mir ein Weib verirrt,

dann ist die Hölle weltverwirrt.«

und

»Mein Geschlecht schimpft mich Verräter

ich bin der Albtraum aller Väter.«

»Ich stehe nicht auf Frauen «, wäre das erste zu übersetzen, und das zweite vielleicht so: »Gemessen an der auf heterosexuelle Fortpflanzung ausgelegten Konstruktion der menschlichen Geschlechtsorgane erscheint meine sexuelle Neigung als Abart; Väter wünschen sich heterosexuelle Söhne.«Ganz geht es nicht auf. »Wenn sich an mir ein Weib verirr"«, klingt bloß altertümlich. Aber was soll die Konsequenz sein: "Wenn mich eine Frau anmacht, dann schlägt's dreizehn / dann steht die Welt Kopf / dann verwirrt sich meine (höllisch unzüchtige) Neigung durch die Konfrontation mit der (heterosexuellen) Welt «? $?^{3}$

Wenig besser verhält es sich mit dem zweiten Verspaar: Dass dem Geschlecht ein personales moralisches Verdikt über seinen Besitzer möglich ist, kann als etwas pathetische Psychologisierung des Körperteils oder Verkörperlichung der Psyche absorbiert werden. Dass Homosexualität der Albtraum aller (konservativ denkender) Väter ist (die ihre Heterosexualität,

3 Dass hier etwas nicht aufgeht, ist kein hermeneutisches Problem, sondern ein hermeneutisches Ergebnis. Die Subtextierung einzelner Passagen soll nicht den Eindruck vermitteln, der ästhetische Gehalt des Textes sei auf einen teilweise mitgedachten (und stets wieder durchkreuzten) Subtext reduzierbar. Im Gegenteil dient die Subtextierung mindestens gleichberechtigt dazu, nicht-substituierbare bzw. nicht-subtextierbare Anteile des Textes freizulegen. 
könnte man forsch vertreten, ja durchs Vatersein unter Beweis gestellt haben), lässt sich nachvollziehen. Dass ein homosexueller Sohn der Albtraum seines (konservativ denkenden) Vaters ist, ebenso. Wer ist aber »ich«, wenn offenbar nur eine Person und doch Albtraum aller Väter?

Diese Aussagen sind nichtsdestotrotz relativ leicht zu entschlüsseln, ebenso die mit ihnen verbundene (Ab-)Wertung. Hier werden die (extrem konservativen) Wertmaßstäbe reflektiert, die zu einem Ekelschrei »Schwule! Ah!« führen könnten: Widernatürlichkeit, Reproduktionszwang, Patriarchat. Und doch enthalten sie einen Restbestand - und sei es an reimender Ungeschicklichkeit - , der sich einem vollständigen Aufgehen in dieser dechiffrierten Lesart verweigert.

Durch diese den Refrains vorangehenden Zweizeiler geleitet, erhalten dessen Pseudo-Zitate den Geruch der Doppeldeutigkeit, der sie zu dechiffrieren erlaubt. »Mann gegen Mann« meint also im Klartext »Mann mit Mann«. »Meine Haut gehört dem Herrn« wäre etwas wie »Jeder Zentimeter meines Körpers steht meinem Freund zu sexuellen Diensten«, oder »Der >taktile Ernstfall< (vgl. Seel 2003: 299) von Sexualität ist bei mir Männern vorbehalten.« »Gleich und gleich gesellt sich gern« heißt - einfach ein Spezialfall der ursprünglichen Aussage - »Homo passt zu Homo, Mann zu Mann«. »Ich bin der Diener zweier Herrn« könnte meinen: »Ich habe zwei Freunde und bevorzuge mit innen Praktiken sexueller Unterwerfung «, aber auch eine Zote wie »Ich habe meinen Freund, und ich habe meinen kleinen Freund«. Und doch: »Meine Haut gehört dem Herrn« klingt so biblisch, ist es biblisch? »Ich bin der Diener zweier Herrn« reimt sich hölzern im gleichen Wort, soll das auch Bibel sein oder doch Carlo Goldoni? Und »Mann gegen Mann«, der titelgebende Ausruf, lebt von dem Spannungsverhältnis, zugleich militärischer Schlachtruf und homosexueller Lustschrei zu sein. Ein archaisches Männerbild - Kampf eines jeden gegen jeden, entfesselte und auf sich gestellte männliche Urgewalt - schreibt er der homosexuellen Vereinigung ein.

Was Wertung betrifft, ist diese Kategorie von Textstellen schon weit genug von den konkreten Tatbeständen auf Anspielung zurückgezogen, um neutral zu wirken. »Gleich und gleich gesellt sich gern« enthält eine abwertende Konnotation dahingehend, dass es im alltagssprachlichen Gebrauch u.U. aus gutbürgerlicher Perspektive auf >Gesindel < gemünzt ist. Der Affizierung mit dem Gleich und Gleich homosexueller Liebe gelingt aber immerhin der Coup, das schwule Prinzip einem wertkonservativen gesellschaftlichen Gemeinplatz einzuverleiben, dessen Vorbehalte gegenüber Schwulsein sozusagen mit den eigenen argumentativen Waffen zu schlagen. Auf Ähnliches könnte man bei der Phrase »Mann gegen Mann« plädieren: 
Einer archaisch-militaristischen, durchaus homophoben Umgebung wird das Schwule wie ein Kuckucksei in die Formulierung gelegt. Auch der Einschluss des »Herrn« als Bezugspunkt schwul-sexueller Subordination mag als Blasphemie Gegenstand neuerlicher Empörung sein, stattet aber zugleich die schwulen Sexpartner mit religiöser Dignität aus. Am Ehesten noch ließe sich an der Wendung, Diener zweier Herrn zu sein, das schizophrene Doppelleben ablesen, einer schwulen Neigung genauso wie einer ihr konträren Gesellschaft >dienen< zu müssen.

Die am wenigsten explizite, die diffuseste Kategorie, man ahnt es, ist die gegenüber Homosexualität affirmativste, und sie findet sich innerhalb der Strophen, bestreitet somit den mit Abstand umfangreichsten Anteil des textlichen Geschehens. Drei vierzeilige Strophen und eine nach zwei Zeilen abbrechende vierte Strophe entwerfen surreale Bilder ungestörter schwuler Utopien, so subtil, dass sie als schwul kaum mehr zu erkennen sind. In der ersten Strophe erscheint die schwule Neigung als weltabgewandter Glücksfall:

»Das Schicksal hat mich angelacht

und mir ein Geschenk gemacht, warf mich auf einen warmen Stern, der Haut so nah, dem Auge fern.«

Ein »warmer Stern« ist natürlich auffällig, weil der stellare Raum eher mit Kälte assoziiert ist. Ein Stern selbst (im korrekten astronomischen Sinne) wäre wiederum (zu) heiß. Zu denken ist also an einen warmen Planeten. Mit »Haut« und »nah« geht dessen Wärme zusammen, nicht aber dessen buchstäblich extraterrestrische Entfernung, die »dem Auge fern« sein muss, nicht aber »der Haut so nah« sein kann. So vereinigen sich Wärme, Haut und taktile Nähe zu einer Projektion der Ferne vor der Welt und ihrem optischen Zugriff. (Oder sollte die Erde, der hiesige warme Planet, gemeint sein?)

Der folgende Zweizeiler ist expliziter, was gerechtfertigt hätte, ihn bereits im Zuge der oben besprochenen, die Refrains anbahnenden Zweizeiler zu besprechen.

»Ich nehm' mein Schicksal in die Hand, mein Verlangen ist bemannt.«

Was er mit den Strophen gemeinsam hat und was ihn von den Zweizeilern unterscheidet (abgesehen davon, dass auf inn kein Refrain, sondern die zweite Strophe folgt), ist die positive Einstellung zur Homosexualität. Das in der ersten Strophe eingeführte »Schicksal« wird offensiv angenommen. Explizit im Sinne der Aphorismen des Refrains ist das insofern, als die Formulierung doppeldeutig ist: »Ich nehm' mein Schicksal in die Hand « heißt 
»Ich steh dazu«, aber auch »Ich masturbiere«. »Mein Verlangen ist bemannt « ist unschwer zu verstehen als »Mein Verlangen richtet sich auf Männer «, knüpft aber über die Assoziation mit einem bemannten Raumfahrzeug an das utopische Bildspektrum des »warmen Stern[s]« aus der ersten Strophe an.

Auch die zweite Strophe verlegt sich auf die planetarische Metaphorik, indirekt zumindest:

»Wo das süße Wasser stirbt, weil es sich im Salz verliert, trag ich den kleinen Prinz im Sinn, ein König ohne Königin.«

Denn der kleine Prinz lebt bekanntlich auf einem sehr kleinen Planeten, dessen Herrscher er gewissermaßen ist. Eine Königin gibt es dort nicht, es sei denn, man rechnet die Rose hierzu: Ausgerechnet zu einer Rose - um deretwillen im nächsten Titel des Albums Rosenrot (2005) eine Frau verderbliche Macht über einen Knaben ausüben wird - unterhält der kleine Prinz auf seinem »warmen Stern« ein Verhältnis fürsorglicher Hörigkeit (vgl. Saint-Exupéry 1950), »was sie will, bekommt sie auch« (»Rosenrot«). Auf die sexuelle Ebene übertragen, kommt der »kleine Prinz « alias das Geschlechtsteil des lyrischen Ichs mit sich selbst aus, ohne von weiblicher Geschlechtlichkeit regiert zu werden. ${ }^{4}$ Im Bild der Mündung eines (Süßwasser-)Flusses ins (salzige) Meer wird die Reinheit des männlichen Geschlechts in dieser Strophe physiologisch: als Reinheit seines Sekrets. Das ist eine direkte Gegenposition zum angeblichen Verrat der Homosexualität an der Bestimmung des Geschlechts, dessen das lyrische Ich im späteren Zweizeiler bezichtigt wird (»Mein Geschlecht schimpft mich Verräter«, s.o.). Hier ist das vermeintlich naturgegebene Heterosexuelle das Lebensbedrohliche, welches Süße sinnlos verwässert bzw. versalzt.

Die dritte Strophe entzieht sich für mich einer metaphorischen Auflösung. Ich verstehe nicht, was die ersten beiden Verse heißen könnten, aber mir gefällt das schiefe, abstrakte Bild, das in irgendeiner Form in den Ausdruck von Wohlbefinden und Harmonie hineinpasst, den die Strophe insgesamt vermittelt:

»Ich bin die Ecke aller Räume, ich bin der Schatten aller Bäume. In meiner Kette fehlt kein Glied, wenn die Lust von hinten zieht.«

4 Vgl. im vorigen Titel »Benzin «: »Brauch keine Frau, nur Vaselin «. 
Die zweite Hälfte bietet wieder mehr Anhaltspunkte: »Glied « ist, gewohnt doppeldeutig, einerseits Bestandteil einer »Kette«, andererseits Geschlechtsteil. Die von hinten ziehende Lust verweist auf einschlägige Sexpraktiken. Es könnte also, mit Verlaub, heißen: »Alle meine Sexpartner sind da, wenn wir Lust auf Analsex haben.« Auch eine Assoziation der aus mehreren Gliedern zusammengesetzten Kette mit entsprechenden Sexspielzeugen ist denkbar.

Die vierte Strophe teilt sich ihre zweite Hälfte mit dem Ende des zweiten Refrains, der den Ausruf »Schwule! « herbeiführt. Insofern lässt sich über die Bewertung als >Strophe d durchaus streiten. Die genuine erste Hälfte passt zur Welt aus Wärme und Wohlbefinden. Die fremde zweite Hälfte, schon auf »Schwule! « hinsteuernd, kommt dementsprechend mit Kälte daher:

»Mich int'ressiert kein Gleichgewicht, mir scheint die Sonne ins Gesicht.

Doch friert mein Herz an manchen Tagen.

Kalte Zungen, die da schlagen:

Schwule!«

Kälte und Herz (und Sonne) ist eine Rammstein-typische Verbindung. ${ }^{5}$ Im letzten Vers wird die alltagssprachliche Wendung von »bösen Zungen« dem Kältetopos (»kalte Zungen«) und die von »bösen Zungen, die da sagen«, dem Gewalttopos (»die da schlagen«) gefügig gemacht, so scheint es: Der Ausruf »Schwule! « wird dadurch (metaphorisch oder real verstanden) zu einem Akt körperlicher Brutalität.

Ist »Mann gegen Mann« Schwulen-Idyll oder Schwulen-Hetze? Der textgestützte Befund deutet darauf hin, dass konträre Meinungsäußerungen unterschiedlicher Explizität und Diffusität in Szene gesetzt werden, teils in klar zugeordneten Textgliedern, teils auf der Mikroebene von Doppeldeutigkeiten und Metaphern. Die berechtigte Frage, inwieweit die Bewertung als mehr oder weniger explizit in jedem Fall mit objektiver Sicherheit vollzogen werden kann, und inwieweit nicht auch strukturelle Beobachtungen oder die Kenntnis von Musik und Video verfälschend miteinfließen, ist doch losgelöst von der zentralen Erkenntnis: Es gibt stark unterschiedliche Grade an Konkretheit und Diffusität auf der einen und an Affirmation und Ablehnung auf der anderen Seite.

5 Vgl. z.B. "Sonne« (Rammstein 2001): »Wenn sie [die Sonne] aus den Fäusten bricht, legt sich Eis auf das Gesicht, legt sich schmerzend auf die Brust, das Gleichgewicht wird zum Verlust.« 
- Die vier Strophen (die vierte nur zur Hälfte) entwerfen das Bild einer schwulen Utopie in subtilen bis abstrakt-kryptischen Bildfeldern. Wärme, Nähe, Haut, Weltabgewandtheit, Harmonie, Vollständigkeit sind hier die Schlagwörter.

- Die Refrain-Teile operieren mit alltagssprachlichen Versatzstücken und Pseudo-Zitaten und inszenieren sie in geschickter Doppelbödigkeit, die die Ambivalenz der Bewertung des schwulen Prinzips erhält.

- Der schwule Kontext wird vermittelt durch drei Zweizeiler, die in zwei Fällen dem Refrain vorangehen: Ihre Statements sind ziemlich leicht auf Homosexualität zu dechiffrieren, wenn auch ein Restbestand an unbegradigter metaphorischer Schiefwinkligkeit bleibt. In zwei von drei Fällen geben sie negative Sichtweisen auf Homosexualität wider.

- Das Kind beim Namen genannt wird in dem wiederkehrenden und am Ende mit der Refrain-Zeile »Mann gegen Mann« alternierenden Ausruf »Schwule! « Durch den Zusatz »Ah!« ist die Verfemung in unartikuliertem Ekel auf die Spitze getrieben.

\subsection{Musik}

Dem ganzen Stück liegt, von wenigen Ausnahmen abgesehen, folgendes Riff zugrunde, das sich durch einen charakteristischen Kleinsexten-Sprung am Anfang und einen retardierend synkopischen Vier-in-Drei-Rhythmus auszeichnet:

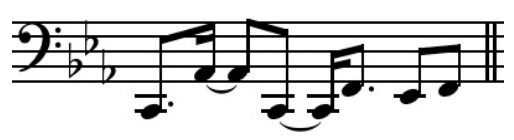

Beispiel 1: Rammstein: »Mann gegen Mann«, Bassriff.

Es wird erst im Synthesizer vorgestellt, bis es dann, nach dem Einzählen der $\mathrm{Hi}$-Hat, in den unverzerrten E-Bass wechselt. Dort läuft es während der Strophen außer der vierten (s.u.) durch. Zum Refrain verändert sich nicht die Struktur, wohl aber der Sound: Nun wummern die Gitarren elektrisch verzerrt, wobei der Klang durch parallele Quinten (Powerchords) an Masse gewinnt.

In den zweiten Refrain ist ein Zwischenspiel (Bridge) eingefügt. Es bringt ein anderes, stark repetitives Riff, das von einer 3-gegen-4-Rhythmik aufgepeitscht ist. Die gleichzeitige Synthesizer-Melodie ist intervallisch mit dem Anfangs-Riff verwandt: Dem charakteristischen kleinen Sextsprung $g^{2}$-es 
entspricht ${ }_{1} C-{ }_{1} A s$ im Riff, andererseits ist das im zweiten Takt erreichte $a s^{2}$ die kleine Sexte über dem Grundton, entsprechend dem ${ }_{1} A s$ über ${ }_{1} C$ im Riff. Die Synthesizer-Melodie operiert auf der Quinte $g$ statt auf dem Grundton:

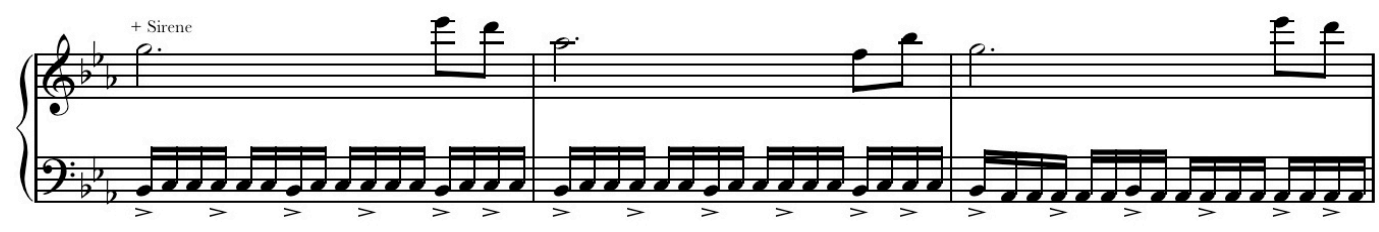

Beispiel 2: Rammstein: »Mann gegen Mann«, Zwischenspiel, T. 48-50.

Dieselbe Melodie liegt wiederum in der Bassgitarre der unverzerrten und schlagzeugfreien zweiten Hälfte des Zwischenspiels zugrunde. Dadurch geraten die Quinte und kleine Sexte in den Bass, die harmonische Szenerie wirkt dominantisch-subdominantisch. Entsprechend artikuliert die Begleitung der oberen Gitarrenstimme gebrochene $D^{46}$ - bzw. $s^{(3) 56}$-Akkorde. Dies ist die Grundlage für die vierte Strophe (»Mich int'ressiert kein Gleichgewicht...«), die sich damit radikal von allem anderen Geschehen abhebt:

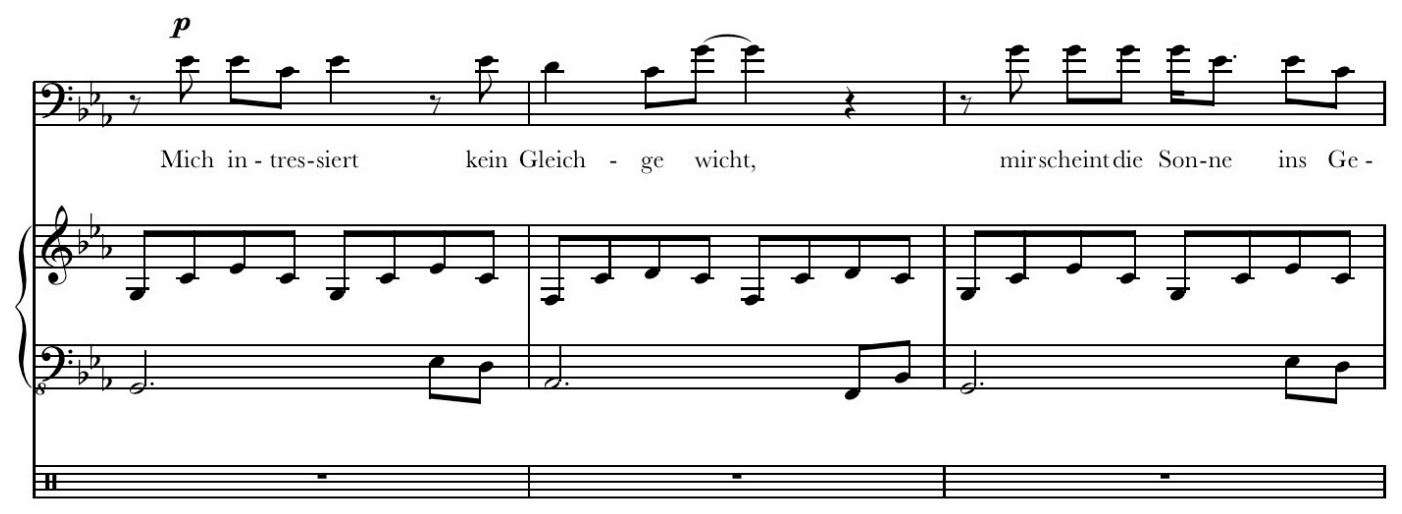

Beispiel 3: Rammstein: »Mann gegen Mann«, T. 64-66.

Die Stimme folgt rhythmisch weitgehend dem leicht aufgerauten Sprachrhythmus. Es gibt nur drei signifikante Abweichungen: Jeweils der Abschluss des letzten Verses einer Strophe ist eigentümlich gedehnt. Till Lindemann gibt sich gar keine Mühe, der verlängerten Phrase das nötige Mehr an Spannkraft sängerisch einzugeben, er atmet mitten zwischen die auseinandergezogenen Silben der Wörter: 

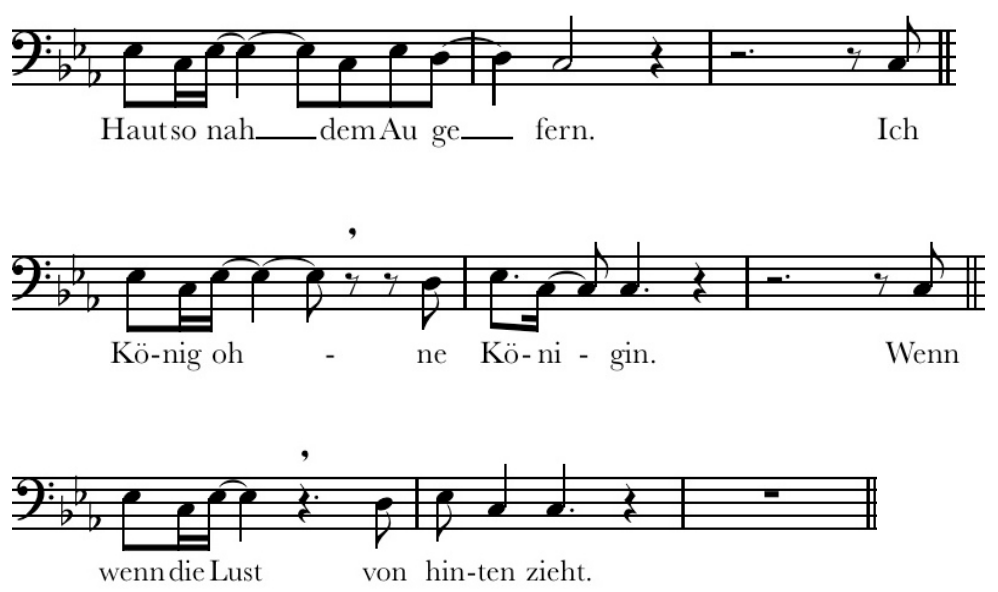

Beispiel 4: Rammstein: »Mann gegen Mann«, T. 7-9, 17-19 und 35-37.

Während in den Strophen ein erzählend beschwörender, man möchte fast sagen, verträumter Stimmklang und eine entsprechende Deklamation vorherrschen, wird der Ton während der in gesteigerter Konkretheit zum Refrain überleitenden Zweizeiler ruppiger, rollender (Prechorus). Das gilt auch für den ersten Zweizeiler zwischen erster und zweiter Strophe, auf den in der Textvorlage gar kein Refrain folgt (»Ich nehm mein Schicksal in die Hand...«) und der aufgrund seiner positiven Einstellung zu Homosexualität einen Zwischenstatus inne hat (s.o.). Mit Kenntnis der Musik sieht es anders aus: $\mathrm{Er}$ ist musikalisch klar mit den späteren beiden Zweizeilern verbunden, die den ersten und zweiten Refrain herbeiführen (»Wenn sich an mir ein Weib verirrt...« und »Mein Geschlecht schimpft mich Verräter...«). Auf inn folgt zwar kein Refrain-Text, wohl aber Refrain-Musik: das Riff mit wummerndem E-Gitarren-Sound. Es ist ein nur zweitaktiges Aufbrechen, das gleich wieder in den (instrumental) unverzerrten bzw. (stimmlich) beschwörenden Duktus der Strophen zurückgeholt wird. Formal ist es von entscheidender Bedeutung: Mit dem Verzicht auf den Refrain-Text und die Beschränkung auf nur zwei Takte hält es den textlich-inhaltlichen Fortgang der ersten beiden Strophen nicht auf. Das Stück soll zunächst einmal textlichinhaltlich vom Fleck gekommen sein, bevor es sich auf die Schlag auf Schlag, Vers gegen Vers hingeworfenen Versatzstücke des Refrains einschießt. Zugleich künden diese zwei Takte zu einem frühen Zeitpunkt von der brutalen musikalischen Gegenwelt, die die später ausgeführten und anteilig überhand nehmenden Refrains bringen werden.

Samples finden sich in den Strophen und verstärken das zum Kitsch hin Offene an ihnen: Zum einen zweimal eine Art synthetisches Kinderlachen, das zugleich >schwul < und wie eine Äußerung von Ekel oder Auslachen klingt, zum anderen Synthesizer-Streicher, mit dem oben erwähnten Chimes- 
Sample im Einschwingvorgang, die die Strophen nach den gedehnten vierten Versen ausläuten. Derselbe Sound findet sich bei 1'21", um den Refrain während der Dauer von nur zwei Takten in die subtile Welt der dritten Strophe zurückzuholen (hier setzt das Riff für zwei Takte aus).

Die Musik scheint die oben vorgenommene Abwägung der Textteile hinsichtlich ihres Grades an Explizitheit durch einschlägige Korrespondenzen zu unterstützen. Der textlichen Differenzierung nach >explizit - verschlüsselt bzw. shomophob - homophil entspricht eine klangliche Orientierung nach shart - weich < und >laut - leise<. Die graduellen und mehrstufigen Unterschiede der Textglieder ordnen sich in Verbindung mit dem musikalischen Material zu stärker binären Gegenüberstellungen.

- In der Musik findet sich eine deutliche Polarisierung zwischen den fragilen, transparenten Strophen- und den massiven, brutalen Refrain-Teilen. Sie ist klanglich (unverzerrt vs. verzerrt), nicht durch musikalische Strukturen vermittelt (das Anfangs-Riff ist beiden ohne strukturelle Veränderung eigen).

- $\quad$ Die erste Hälfte der vierten Strophe wird durch eine ganz andere musikalische Textur herausgehoben (die sie in ihren Elementen allerdings mit dem vorangegangenen Zwischenspiel teilt). Sie wird zur fragilsten Passage des ganzen Stücks.

- Die Zitat-ähnlichen Äußerungen des Refrains (»Gleich und gleich gesellt sich gern« etc.) werden durch die musikalische Umsetzung der Seite des harten, massiven Klangs zugeschlagen.

- Die drei Zweizeiler (»Ich nehm mein Schicksal in die Hand...«, »Wenn sich an mir ein Weib verirrt...«, »Mein Geschlecht schimpft mich Verräter...«) haben als Prechorus klar überleitende Funktion vom Strophenin den Refrainsound. Der Übergang vollzieht sich über den sich verhärtenden Stimmklang und die ausbruchsartig in den Refrain stürzende Schlagzeug-Aktivität.

- Die Sonderstellung der Wendung »Mann gegen Mann«, die den anderen Zitaten sowie dem Ausruf »Schwule! « beharrlich entgegengesetzt wird, wird musikalisch genutzt, insofern der Ausruf als einziger nicht tonlich, sondern dumpf gegrowlt von allen anderen Versen abgegrenzt wird. »Mann gegen Mann! und »Schwule!«, die beiden durch Wiederholung insistierenden Elemente, werden so in ihrer kontrapunktischen Stellung verschärft. 


\subsection{Video}

Es gibt »narrative Clips « und es gibt Clips, »die eher als Performance und mithin wenig bzw. gar nicht erzählend konzipiert sind«, sog. situative Clips (Keazor/Wübbena 2007: 27, vgl. zur Kategorisierung ausführlich Altrogge 2001). Da der hier zugrunde liegende Text nicht erzählerisch ist, verwundert es nicht, dass man im zugehörigen Clip einen Vertreter der letzteren Kategorie antrifft. Die Band wird beim Spielen des Songs gezeigt. Das ist auf einer vordergründigen Ebene schon fast alles; einzig ein radikaler Ausbruch aus dieser eher unnarrativen und statischen Umgebung entfaltet gegen Ende des Videos umso ungeheurere Wirkung.

Paul Landers beschreibt die »Story« so:

»Die Story ist, wir spielen nackig, komplett, wobei die Gitarristen jetzt die Gitarre davor haben, das ist 'n leichterer Job. Till hat sich dann 'ne Lackwindel angezogen, weil das für ihn doch nichts war, und alle anderen sind komplett nackig« (zit. n. Fuchs-Gamböck/Schatz 2010: 155).

Die mise-en-scène, das filmisch Dargestellte, kennt in diesem Clip zwei Umgebungen. Die eine ist für die weitaus längste Dauer des Videos konstant, sodass sie >Grundumgebung - genannt werden kann. Ihr prägnantestes Ausstattungsmerkmal ist ein rosaroter Teppichboden, der von einer Scheinwerferbrücke von oben angestrahlt wird. Wände oder andere räumliche Begrenzungen sind aufgrund der Lichtverhältnisse nicht zu erkennen, genauso wenig irgendeine Form von Mobiliar. ${ }^{6}$

In dieser Umgebung spielen sich zwei voneinander getrennte Handlungen vor der Kamera ab. Die eine zeigt die Bandmitglieder beim Spielen des Songs. Schwarze Kabel winden sich auf dem rosafarbenen Boden und lassen ihn als Studio- oder Bühnenraum erscheinen. Niemand hat, von (Springer-) Stiefeln abgesehen, etwas an außer Frontmann Till Lindemann, dessen Blöße statt durch ein Instrument durch eine »Lackwindel« (zit. n. ebd.: 155) bedeckt wird. Außerdem trägt er kniehohe Lackstiefel, mit Pfennigabsatz. In Abweichung von seinem gewöhnlichen Äußeren sind seine Haare überschulterlang. Sein leicht aufgedunsener Körper ist behaart. Die Physiognomie ist grau, schweißnass, Augen und Mund meist spastisch aufgerissen. Schön sieht er so nicht aus. Schön aber sehen sie aus in der parallelen Handlung: nackte Athleten, schwarze und weiße, mit ungeheuerlichen, ölglänzenden Körpern. Zur ersten Strophe lassen sie ihre symmetrisch durchtrainierten, schimmernden Gliedmaßen in choreographierten Bewegungen

6 Vgl. zur hier durchgeführten Musikvideoclip-Analyse Jost 2009. 
spielen. Als gäbe die Musik mit dem ersten, nur zweitaktig angetäuschten Einsatz des Refrains ein Signal, zuckt, als die E-Gitarren loswummern, ein gigantischer Brustmuskel. Ab dann umschlingen und berühren sich die verschiedenfarbigen Körper mit zärtlich festem Druck, kauern, liegen, zu vielarmig wogenden Arrangements verknotet, auf dem rosafarbenen Boden, ein lebendig ineinander verschlungener Organismus.

An ihrer (identischen) räumlichen Umgebung lassen sich beide Geschehen nicht unterscheiden und auch am Personal nur schwer, denn auch wenn die nacktglänzenden Musiker keine Athleten sind, fällt nur der behaarte und graufahle Till Lindemann offensichtlich aus dem Rahmen. Es ist die mise-enchaîne, die eine klare Aufgabenteilung vornimmt: Die musizierende Band wird in Naheinstellungen hauptsächlich auf die Gesichter fokussiert; auch in Halb- oder Ganzkörperaufnahmen und verschiedenen Totalen (von vorne, von der Seite) sind die Einzelpersonen stets an ihrem Gesicht und Instrument zu erkennen. Anders die Athletenkörper: Innen sind fast ausschließlich Nahaufnahmen vorbehalten: Brustmuskeln, Sixpacks, Bizepse, Schulterblätter, Hände. Die wenigen Totalen lassen Rücken und Hinterteile sehen, aber nicht ein Gesicht eines Athleten tritt so in Erscheinung, dass es wiedererkennbar wäre. Sie sind nur Körper, Körperteile vielmehr, und als solche so gefilmt, dass sie oft nicht einmal einzelnen Körpern zugeordnet werden können. Die Art, wie diese Auflösung und Verschmelzung der Körper zu neuen, die zur Verfügung stehende Bildfläche kompositorisch gestaltenden Formationen vor sich geht, erinnert an die körperbetonte Ästhetik Leni Riefenstahls. $^{7}$

Ihre je klare filmische Akzentuierung trägt indes nicht dazu bei, dass beide Ebenen zu jeder Zeit tatsächlich klar unterscheidbar wären. Bisweilen scheint es, als hätten sich die Körper der Musiker vervielfältigt und seien

7 Zu ihrer Cover-Version des Depeche-Mode-Titels »Stripped« (1998) verwendete Rammstein Montagen aus Leni Riefenstahls Film Fest der Völker / Fest der Schönheit über die Berliner Olympischen Spiele 1936. In Verbindung mit den »martialischen Live-Auftritte[n], ein Spiel mit Muskeln und Feuer, begleitet von einer Art teutonisch-brachialem Metal« führte das zu einer heftigen medialen Kontroverse. Rammstein gab später an, mit der Montage eine Grenze überschritten zu haben, was nicht wieder vorkommen werde (Fuchs-Gamböck/Schatz 2010: 103). In eigenen Statements wird differenziert zwischen der ästhetischen Befürwortung Riefenstahls und ihrer politischen Bedeutung: Man finde sie »künstlerisch gut«, sei aber deswegen »doch kein Nazi«. Generell dementiert Lindemann »faschistoide Ästhetik « bei Rammstein: »Absoluter Schwachsinn. Es werde verkannt, dass es sich »bei den Shows um eine Form von Theater « handele (Lindemann 2001: 33). Dieser Zusatz erlaubt indes, auch dieses Dementi auf die politische Bedeutung sfaschistoider Elemente zu beziehen, von der Rammstein losgelöst sein will, nicht aber auf deren ästhetische Dimension, die in einem Kunstraum theatraler Inszenierung sehr wohl stattfindet. 
dieselben, die sich da gesichtslos lustvoll aneinander rieben. Dazu trägt die schnelle Schnitt-Technik bei, die die parallelen Ebenen teilweise im Sekundentakt miteinander verschneidet. Der Rhythmus der Musik ist hierbei einzig bestimmendes Element. Besonders augenfällig ist die Koppelung bei dem Schlachtruf »Mann gegen Mann«, der stets auf die Silbe »Mann« den zum Schrei aufgerissenen Mund eines der Bandmitglieder (meist Till Lindemanns) buchstäblich anspringt. Teilweise wird noch in Sekundenbruchteilen nachgezoomt, oder das Gesicht platzt seitlich versetzt und zu groß auf den Bildschirm.

Das Beschriebene ist der >Grundzustand des Videoclips, die >Grundumgebung ( (die rosa Bühne), die >Grundhandlung ( die musizierende Band, die sich als narzisstischer Super-Organismus gerierenden Athletenkörper). Folgende Abweichungen geschehen:

- Bei 1'21" fängt ein Synthesizer-Streicher-Akkord die Wucht des Refrains in nur zwei Takten ab, um in die fragile dritte Strophe zu führen. Anzumerken ist hier eine Detailbeobachtung der mise-en-scène: Der letzte Schlag von Gitarrist Paul Landers läuft in eine kreisende Bewegung des ausgestreckten Arms aus, die zum Verwechseln einem Hitlergruß ähnelt. Während der dritten Strophe wird mit weiteren, den Spielbewegungen entlehnten Gesten experimentiert, etwa dem periodischen Anschlagen der Saiten durch Hin- und Herschwingen des ganzen Arms. Der Position der Gitarre vor dem Unterleib entsprechend hat die Bewegung etwas Obszönes. Die damit verbundenen Konnotationen mögen kalkuliert oder versehentlich entstanden sein: Das Rezeptionsangebot besteht für jeden entsprechend sensibilisierten Betrachter. ${ }^{8}$

- Diese zwei Takte nach 1'21" haben musikalisch eine besondere Stellung, da in ihnen zum einzigen Mal während des ganzen Stücks das GitarrenRiff pausiert. Hier hält die Musik freischwebend inne, auch wenn die Timeline im Hi-Hat über die zwei Takte weiterläuft. Der Film trägt diesem musikalischen Umstand höchst subtil Rechnung, auf der Ebene der mise-en-cadre. Die Montagefrequenz ist nur wenig reduziert, entscheidend ist, dass für die Dauer dieser zwei Takte die Schnitte als Überblendungen stattfinden. Mit Beginn der dritten Strophe folgen wieder die üblichen harten Schnitte.

- Während des nachfolgenden Refrains, etwa ab 1'57", meint man eine allmähliche Radikalisierung der wogenden Athletenhaufen zu erkennen.

8 Es ist nochmals zu betonen: Hier geht es nicht um die Spekulation über Intuitionen oder Gesinnungen der Band, sondern um das, was sich der Rezeption darbietet. 
Mehr und mehr werden Körper und Extremitäten in geworfener oder taumelnder Bewegung gezeigt, während die flächigen Berührungen zwischen den Körpern weniger werden.

- Synchron mit dem Beginn des Zwischenspiels (2'13") setzt feiner, im Scheinwerferlicht gleißender Sprühregen ein. Er sprenkelt offenbar aus Düsen in der unsichtbaren Decke der Halle, die sich genau oberhalb der Position eines jeden der Musiker befinden. (Auch die Athleten ringen nun in der Nässe.) Das führt zu einer erheblichen Eskalation des Verhaltens der Musiker. Ihre Bewegungen werden zuckend, spastisch, epileptisch, mit verdrehten Augen lassen sie sich Wasser in den Mund regnen. Diese durch den Regen ausgelöste Eskalation reicht gewissermaßen das missing link nach zwischen dem offenen Ende des Refrains (»kalte Zungen, die da schlagen «) und seiner Wiederholung und Beantwortung, für die die Aggression erst nach der ersten Hälfte des Zwischenspiels ausreicht (»kalte Zungen, die da schlagen: Schwule! Ah!«). Bei den krampfartig dargebotenen Schreien scheint es, als würde dem mit seinem Lackstiefel nach der Kamera tretenden Till Lindemann für einen Augenblick eine graue Doppelzunge aus dem Mund schießen.

Wie im Übergang zur dritten Strophe wird die Wucht auch zur zweiten, leisen Hälfte des Zwischenspiels (2'48") quasi schlagartig zurückgenommen. Diesmal ist es das Schlagzeug, das acht Takte aussetzt - auch das eine singuläre Erscheinung im gesamten Stück. Erneut trägt dem die mise-encadre Rechnung, indem das ganze leise Zwischenspiel wieder mit Überblendungen anstelle der harten Schnitte bestritten wird. Diesmal gilt der Ausnahmezustand aber für alle Parameter. Es geschieht viel mehr, es geschieht Ungeheuerliches: Eine weiße Hand findet in einer schwarzen Halt. Die Musiker, einer nach dem anderen, werden von der Menge der Athleten getragen, willenlos, wie benommen. Die beiden Hemisphären und ihr Personal kreuzen sich in einem filmischen Raum. Die Anspielung an die auf Rammstein-Konzerten gängige Praxis des crowdsurfing öffnet zugleich die dargestellte homoerotische Harmonie hin zu der realen homosozialen Konzertsituation, in der die männlichen Bandmitglieder von einem vorwiegend männlichen Publikum gefeiert, geliebt und getragen werden. Die mise-enchaîne blickt auf all das von oben herab. Dann wird die >Grundumgebung gesprengt. Ruhigen, verklärten Blicks nach oben aus glasigen und geröteten Augen singt Till Lindemann in eine Schwärze, die nicht mehr wie ein unsichtbarer Bühnenraum, sondern wie freier Nachthimmel aussieht, aus dem statt Sprinklergischt feine Schneeflocken zu fallen scheinen. Lindemann ist auch nicht mehr nackt oder sieht zumindest in dem Nachtlicht nicht mehr so 
aus, gerahmt ist sein Oberkörper von einem graubraunen, rauhen Etwas, das wie Baumrinde, Panzer und Engelsflügel aussieht und auf dem sich rote Blumen abheben (keine Rosen). Er sieht nun selbst aus wie ein abenteuerlich gepanzertes, in einer Phantasielandschaft festgewachsenes Fabelwesen. $\mathrm{Zu}$ seinen Füßen wogen die nackten Leiber und greifen nach seinem im Dunkel unkenntlichen >Sockel<. Man ist versucht, an die Welt des Kleinen Prinzen zu denken, so extramundial mutet die Szene an. ${ }^{9}$

Sie hält nur vier Takte an. Wo Text und Instrumentation das Zwischenspiel in die harte Klanglichkeit des Refrains zurückreißen (3'10"), fährt ein Springerstiefel auf den rosafarbigen Teppichboden nieder und wirft das Geschehen wieder in die >Grundumgebung zurück. Aber wie hat sie sich verändert: Die Leiber ducken sich unter dem niederprasselndem Regen und unter Schlägen, die die Gischt von den Gliedmaßen aufspringen lassen (wie von Becken und Schlagzeug). Till Lindemann reißt sich, »Ah!« brüllend, mit beiden Händen die schwarzen Haare vom Kopf, schwarzgrau sein ganzer Leib, und so entstellt erkennt man in den letzten Sekunden in ihm den vertrauten, kurzhaarigen Till Lindemann.

Was ist die Strategie dieses Films im Verhältnis zu Text und Musik? Er verzichtet auf vieles. Er erzählt keine Geschichte. Er reduziert seinen Vorrat an Bildern und Handlungen auf zwei Elemente: nackte Musiker, nackte Athleten. Er würdigt nicht jeden Bruch in Text und Musik mit einer Variation seiner Parameter: die Grundumgebung, die beide Grundhandlungen, der Grundmodus ihrer filmischen Darstellung und die Montage bleiben über weite Strecken konstant. Nur mit einer subtilen Modifikation der SchnittTechnik nuanciert der Film die beiden Stellen, in denen eine Unwucht musikalischer Energie aus dem Refrain bzw. aus der ersten Hälfte des Zwischenspiels binnen Sekunden abgefedert wird. Umso dramatischer dann dieses eine Heraustreten, dieses plötzliche Geworfensein in eine (scheinbar) andere Welt.

Bis in seine Binnenstruktur verlässt sich der Film auf den Rhythmus der Musik. Das wiederkehrende »Mann gegen Mann« gewinnt durch seine konsequente Akzentuierung im Schnitt noch mehr Gewicht, als ihm durch seinen textlichen und klanglichen Sonderstatus schon zukommt.

Der Film macht was aus diesem »Mann gegen Mann«. Der militaristische bis faschistoide Beigeschmack wird geschärft und erweitert: um rassischen (wenn auch inter-rassischen) Körperkult, um Leni-Riefenstahl-Ästhetik, um (versehentlichen) Hitlergruß, um Rosa-Farbenes erschütternden Springerstiefel, um Gewaltdarstellung in Zeitlupe, in der die kraftstrotzende homo-

9 Die Szene ist auch auf dem Cover der Single zu sehen. 
erotische Mannesschönheit zugleich untergeht und erst gischtsprühend sich vollendet. Das Schwule daran ist von vornherein mit dem ihm Opponenten kontaminiert. Der Springerstiefel, der so scheinbar von außen auf den Teppich niedersaust, verweist mitten ins Herz seiner rosaroten Gegenwelt. Gewalt und Aggression, und zielt sie auch auf Selbstzerstörung, sind tief diesem homoerotischen Idyll eingesenkt. So martialisch wie es sich zelebriert, hält es sich selbst den Spiegel der Entartung vor.

Till Lindemann ist der Gegenentwurf zu solcher bis zur Unkenntlichkeit mit ihrem Gegenteil geratenen Homoerotik. Von Körperkult ist er, der Unförmige, Behaarte, frei. Von der mit jenem Kult paktierenden Gewalt auch, denn der eine Tritt seines Lackstiefels in Richtung der Kamera wirkt, anders als den des anonymen Springerstiefels, eher hektisch als brutal. Seine Insignien des Schwulen sind das Krankhafte, Abartige, Hässliche, und das Lächerliche (Lackstiefel und »Lackwindel«). Bis auf die langen Haare ist er das aus einer klischierten Gegenideologie erwachsene Hohnbild des Schwulen, der buchstäbliche »Albtraum aller Väter «. So pervers wie er sich geriert, hält er der athletischen Gegenwelt den Spiegel des Faschismus vor.

Wo sich die Ästhetik auf die Seite des Homoerotischen schlägt, rückt das Resultat in die Nähe des (ästhetisch) Faschistoiden, mithin (implizit) Homophoben. Wo die Ästhetik auf Solidarisierung mit dem Homoerotischen verzichtet, gibt sie es seiner vermeintlichen Krankhaftigkeit und Perversität preis, im Sinne des (ideologisch) Faschistoiden, Homophoben. Diese chiastische Struktur entspricht interessanterweise Argumentationsfiguren, die Claus/Müller (2010) in ihrer Untersuchung von Homosexualität im rechtsextremen Milieu aufzeigen:

Einerseits sei »männliche Homosexualität im Koordinatensystem der Männlichkeiten stets untergeordnet « und werde »als Affront gegen hegemoniale Männlichkeit verstanden«. Homophobie sei mithin strukturelle Voraussetzung hegemonialer Männlichkeit, insofern sie »geradezu des konstruierten Homosexuellen als Anti-Typus [bedürfe], des effeminierten und daher >unmännlichen< Mannes« (ebd.: 111f.). Im Musikvideo zu »Mann gegen Mann « ist Till Lindemann klar als solcher Anti-Typus markiert und den hypermaskulinen Athleten gegenübergestellt. ${ }^{10}$

Diese Hypermaskulinität könne jedoch unter bestimmten Bedingungen zur Legitimation von Homosexualität dienen. Insbesondere von homosexuellen Rechtsextremen werde Schwulsein in diesem Fall als Streben nach einem Männlichkeitsideal dargestellt. Man stehe auf Kerle, nicht auf Femini-

10 In anderen Musikvideos oder Bühnenshows erfüllt oft Keyboarder Christian >Flake< Lorenz diese Funktion, von der sich der tendenziell hypermaskuline »Männerbund« (Claus/Müller 2010: 111) der Band abhebt. 
nes. Man kämpfe nicht gegen Männer, sondern gegen Unmännlichkeit. Für homosexuelle Rechtsextreme sind »homophobe Abgrenzungen zu empfundener Unmännlichkeit, Schwäche und femininen Attitüden bei Schwulen [...] zentral in der Reflexion ihres eigenen Begehrens« (ebd.: 115).

»Homosexuelle Nazis müssen jedwede Effeminiertheit vermeiden, wollen sie mehr oder minder offen zu ihrer Homosexualität stehen und als >vollwertige Männer anerkannt werden. Gerade in einer Szene, in der klare Geschlechterrollen gelten und viele einen gewaltförmigen und hypermaskulinen Habitus verkörpern, ist es eminent, nicht als >weiblich ‘ oder `tuntig`zu gelten« (ebd.).

Diese Aufwertung des Homoerotischen, geknüpft an die Bedingung von Hypermaskulinität, verkörpern die Athletenkörper des Musikvideos. Eine so vermittelte Toleranz gegenüber einem bestimmten Konzept von Homosexualität darf freilich »nicht mit Akzeptanz gegenüber Homosexualität an sich verwechselt werden« (ebd.: 116). Im Gegenteil hat sie aggressive Intoleranz und Diffamierung aller anderen Formen von Homosexualität zur Voraussetzung. Diese Konfliktlinie wird denn auch im Video inszeniert.

Auf der Strecke bleibt in dem Musikvideo das unbelastet Utopische am Schwulen, das der Text in den Strophen zu entfalten vermochte. Allenfalls tritt es auf in der einen Ausnahmesituation, die der Film durch die vorangegangene radikale Beschränkung seiner Mittel mit besonderer Wirkung ausstatten kann. Die auf Händen getragenen, auf den Athleten crowdsurfenden Bandmitglieder, vor allem aber der auf den (kalten) Stern gezauberte Till Lindemann mit gepanzerten Engelsflügeln sind frei von den Gegenpolen vom martialisch Gewaltsamen vs. krankhaft Verfemten, in die der Film den Rest getrieben hat. Hier und nur hier kann homosoziale und homoerotische Interaktion außerhalb homophober Rahmungen stattfinden, nur hier kann sie als positiv inszeniert werden, ohne sich durch ihre Affirmation zugleich hinterrücks unterwandert zu sehen.

\section{Fazit}

Die vorliegende Analyse von »Mann gegen Mann« hat gezeigt, dass es Voraussetzung ist, seine Bestandteile als Gegenstände von Inszenierung zu verstehen, um seine ästhetischen Wirkungsstrategie würdigen zu können. Die Analyse liefert mithin einen Ansatz, die opponenten Bewertungen Rammsteins, die jeweils im Vertrauen auf Rammsteins unmittelbare Authentizität gegeben werden, in ihrer Gegensätzlichkeit zu integrieren und als Symptome eines komplexen Spiels von primär unauthentischen Inszenie- 
rungen verständlich zu machen. Erst aus dieser kritischen Distanzierung heraus werden der ästhetische Reiz und die provokative Kraft Rammsteins greifbar und konsistent erklärbar.

Gerade aus dem komplexen inszenatorischen Wirkmechanismus kann auf den Impetus des »Ärgermachens« rückgeschlossen werden, der nämlich gar nicht so sehr in einer scheinbar unliebsamen, aber unmittelbar authentischen Meinungsäußerung gründet. Es geht nicht um authentische, im Sinne von: unverfälschte, ehrliche, inhaltsorientierte Meinungsäußerung, Diskussionsbeiträge etc. (vgl. Knaller/Müller 2006: 7/9; Habermas 2003). Vielmehr wird die Aufhebung der authentischen Meinungsäußerung in ein ausdrücklich inszeniertes ästhetisches Wechselspiel zur eigentlichen Provokation. Dass dieses dabei stets mit außerästhetischen Inhalten interagiert, liegt in der Natur der Sache und in der Beschaffenheit von Ästhetik. Rammstein ist die Fähigkeit nicht abzusprechen, gesellschaftliche Diskurse über Tabuthemen anzustoßen oder zu befeuern; genauso wenig ist Rammstein die Fähigkeit zuzusprechen, in ihrem Schaffen die zugehörigen diskursiven Lösungen mitzuliefern. Nicht durch die glaubwürdige Positionierung zu außerästhetischen Inhalten, erst durch deren raffinierte Inszenierung entsteht bei Rammstein Authentizität. Gerade der von Rammstein selbst häufig vorgebrachte Verweis auf die eigene Unauthentizität (vgl. Grossberg 1992), die bloße spielerische Gemachtheit des jeweils >Gemeinten < kann die Authentizität des Gesamten legitimieren. Man könnte auch sagen: Dass jedem Konzept von inszenierter Authentizität oder staged authenticity ein performativer Widerspruch (Knaller/Müller 2006: 8f.) und mithin eine Provokation innewohnt, materialisiert sich an Rammstein in besonderer und konstitutiver Weise.

Diese Authentizität erweist sich mithin als eine Authentizität zweiter Potenz, die mit der Inszenierung unteilbar verschränkt ist. Rammstein ist als Band glaubwürdig, weil sie Unglaubwürdigkeit von vornherein zu ihrem ästhetischen Steckenpferd macht. Umgekehrt wird so deutlich, dass die Provokation Rammsteins in ihrer Struktur eine ästhetische ist, deren machtvolle Wechselwirkung mit außerästhetischen, gesamtgesellschaftlichen Diskursen gleichwohl vor allem aus ihren immanent ästhetischen Grundbedingungen heraus möglich und sinnvoll ist. 


\section{Literatur}

Altrogge, Michael (2001). Tönende Bilder. Interdisziplinäre Studie zu Musik und Bildern und ihrer Bedeutung für Jugendliche, Bd. 2 (Die Musikvideos), Berlin: Vistas.

Bettendorf, Michele (o. J. [2004]). Ursprung Punkszene oder Rammstein hätte es im Westen nie gegeben. 0.0.: Books on Demand.

Claus, Robert / Müller, Yves (2010). »Männliche Homosexualität und Homophobie im Neonazismus. «In: »Was ein rechter Mann ist...«. Männlichkeiten im Rechtsextremismus. Hg. v. Robert Claus, Esther Lehnert und Yves Müller (= RosaLuxemburg-Stiftung Texte 68). Berlin: Karl Dietz, S. 109-126.

Fuchs-Gamböck, Michael / Schatz, Thorsten (2010). Bis das Herz brennt. Die inoffizielle Rammstein Biographie. Königswinter: Heel.

Grossberg, Lawrence (1992): We gotta get out of this place. Popular conservatism and postmodern culture. New York, London: Routledge.

Habermas, Jürgen (2003): »Zur Architektonik der Diskursdifferenzierung. Kleine Replik auf eine große Auseinandersetzung. «In: Reflexion und Verantwortung. Auseinandersetzungen mit Karl-Otto Apel. Hg. v. Dietrich Böhler, Matthias Kettner und Gunnar Skirbekk. Frankfurt/M.: Suhrkamp, S. 44-64.

Hof, Gert (2001). »Vorwort. «In: Rammstein. Hg. von Gert Hof. Berlin: dgv, S. $3 f$.

Keazor, Henry / Wübbena, Thorsten (2007). Video thrills the Radio Star. Musikvideos: Geschichte, Themen, Analyse. Bielefeld: transcript.

Jost, Christopher (2009). »Integrierte Bild-Text-Ton-Analyse. Am Beispiel des Musikclips Californication. «In: Die Bedeutung populärer Musik in audiovisuellen Formaten. Hg. v. Christopher Jost, Klaus Neumann-Braun, Daniel Klug und Axel Schmidt (= Short Cuts $\mid$ Cross Media 1). Baden-Baden: Nomos, S. 197-242.

Knaller, Susanne / Müller, Harro (2006): »Einleitung. Authentizität und kein Ende.« In: Authentizität. Diskussion eines ästhetischen Begriffs. Hg. v. dens. München: Wilhelm Fink, S. 7-16.

Kruspe, Richard Z. (2001). »Rammstein ist eine Katastrophe.«In: Rammstein. Hg. von Gert Hof. Berlin: dgv, S. 16-23.

Landers, Paul (2001). »Rammstein hätte es im Westen nie gegeben.«In: Rammstein. Hg. von Gert Hof. Berlin: dgv, S. 34-39.

Lindemann, Till (2001). »Meine Vorliebe ist die altdeutsche Sprache.«In: Rammstein. Hg. von Gert Hof. Berlin: dgv, S. 31-33.

Saint-Exupéry, Antoine de (1950). Der Kleine Prinz. Übers. v. Grete und Josef Leitgeb. Bad Salzig: Rauch.

Seel, Martin (2003). Ästhetik des Erscheinens. Frankfurt/M.: Suhrkamp.

Schneider, Christoph (2001). »Wo liegt das Problem?« In: Rammstein. Hg. v. Gert Hof. Berlin: dgv 2001, S. 26-29. 


\title{
Audio-/Videographie
}

Rammstein (1998). "Stripped.« CD-Single: Motor Music 044 141-2; Official Video: http: / /www.youtube.com/watch?v=M4SmZkmLRjQ (letzter Zugriff 3.12.2012).

Rammstein (2001). »Sonne. « Auf: Mutter. Motor Music 549 639-2.

Rammstein (2005). Rosenrot. Universal 987 458-8.

Rammstein (2006). »Mann gegen Mann.« CD-Single: Universal 06024 9877550-9; Official Video: http://www.youtube.com/watch?v=JE1lSafSUzc (letzter Zugriff 3.12.2012).

\begin{abstract}
Few other bands rival Rammstein's capacity to polarise opinion, particularly on the question of authenticity and artificiality. Controversies have centred on the issue of whether the band is fascist or apolitical, homophobic or pro-homosexuality, irresponsibly provocative or liberating. This analysis does not aim to decide this question by defining Rammstein's authentic expression of opinions. It would be inappropriate and misleading to search Rammstein's songs for signs of the band's true convictions. By shifting the focus to the question of whether, to what extent and through which specific artistic means the band's work may seem fascist, prohomosexuality etc, at a given moment, this paper shows that an elaborate interplay of inconsistent, equivocal and diffuse quotations, allusions and symbolism constitutes the effective strategy of Rammstein's work. This strategy may have the potential to stimulate social discussions on extra-aesthetic topics, but it is nevertheless of a genuinely aesthetic kind, as is the provocation it may involve. A detailed analysis of the music video »Mann gegen Mann « provides an example of the indissoluble ambiguity of pro-homosexual and homophobic implications as its central aesthetic point at the levels of text, music and video.
\end{abstract}

